Open Access

\title{
Virtual colonoscopy, optical colonoscopy, or fecal occult blood testing for colorectal cancer screening: results of a pilot randomized controlled trial
}

\author{
John J. You ${ }^{1,2^{*}}$, Yudong Liu ${ }^{3}$, John Kirby ${ }^{4}$, Parag Vora ${ }^{5}$ and Paul Moayyedi ${ }^{1}$
}

\begin{abstract}
Background: No head-to-head randomized controlled trials have demonstrated the superiority of one colorectal screening modality over another in reducing colorectal cancer mortality. We conducted a pilot randomized controlled trial of fecal occult blood testing (FOBT), optical colonoscopy (OC), and virtual colonoscopy (VC), to inform the planning of a larger evaluative trial.
\end{abstract}

Methods: Eligible patients (aged 50 to 70) were recruited from five primary care practices in Hamilton, ON, Canada, between March 23, 2010 and August 11, 2010, and randomized 1:1:1 in a parallel design using an automated, centralized telephone service to either FOBT, OC, or VC. To reflect conventional practice, patients received no additional reminders to complete their allocated screening test beyond those received in usual practice. The primary outcome was completion of the assigned screening procedure. Results of the index test and any follow-up investigations were ascertained at 6 months. Participants, caregivers, and outcome assessors were not blinded to group assignment. The trial was stopped early due to lack of ongoing funding.

Results: A total of 198 participants were enrolled, of whom 67 were allocated to FOBT, 66 to OC, and 65 to VC. The allocated screening procedure was completed by $43(64 \%)$ subjects allocated to FOBT (95\% confidence interval [Cl], $52-75 \%), 53$ (80 \%) subjects allocated to OC (95\% Cl, 69-88 \%), and 50 (77\%) subjects allocated to VC (95\% Cl, 65-85\%); because the trial stopped early, we had insufficient statistical power to detect clinically relevant differences in completion rates. During 6 months follow-up, colorectal adenomas were detected in 0 (0 \%) subjects allocated to FOBT, $12(18 \%)$ subjects allocated to OC, and 2 (3\%) subjects allocated to VC. One subject in the OC arm had histological evidence of high-grade dysplasia. No subjects were diagnosed with colorectal cancer.

Conclusions: In this pilot randomized controlled trial of colorectal cancer screening in a primary care setting, 64-80 \% of subjects completed their allocated screening test. These findings may be of value to investigators planning clinical trials to evaluate the effectiveness of colorectal cancer screening.

Trial registration: ClinicalTrials.gov NCT00865527. https:/clinicaltrials.gov/ct2/show/NCT00865527

Keywords: Colonoscopy, Colorectal cancer, Computed tomography colonography, Fecal occult blood testing, pilot study, Prevention, Randomized controlled trial, Screening, Virtual colonoscopy

\footnotetext{
* Correspondence: jyou@mcmaster.ca

'Department of Medicine, McMaster University, 1280 Main Street West, Room

HSC-2C8, Hamilton, Ontario L8S 4K1, Canada

${ }^{2}$ Department of Clinical Epidemiology \& Biostatistics, McMaster University,

1280 Main Street West, Room HSC-2C8, Hamilton, Ontario L8S 4K1, Canada

Full list of author information is available at the end of the article
}

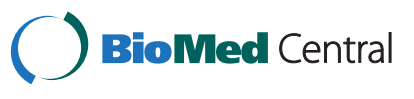

(c) 2015 You et al. This is an Open Access article distributed under the terms of the Creative Commons Attribution License (http://creativecommons.org/licenses/by/4.0), which permits unrestricted use, distribution, and reproduction in any medium, provided the original work is properly credited. The Creative Commons Public Domain Dedication waiver (http://creativecommons.org/publicdomain/zero/1.0/) applies to the data made available in this article, unless otherwise stated. 


\section{Background}

Colorectal cancer is a leading cause of cancer death worldwide, killing an estimated 694,000 people each year [1]. There is evidence that certain colorectal cancer screening modalities may reduce the incidence of adenocarcinoma and that all approaches lead to the detection of earlier stage disease [2]. Current practice guidelines recommend a range of options for colorectal cancer screening, including fecal occult blood testing (FOBT), optical (endoscopic) colonoscopy (OC) and virtual colonoscopy (VC), also known as computed tomography colonography [3, 4]. However, these guidelines do not endorse a single best procedure for colorectal cancer screening, primarily because there are no head-to-head randomized controlled trials demonstrating that one screening test is more effective than another in reducing colorectal cancer mortality. We conducted a pilot randomized controlled trial to estimate the rates of completion of assigned screening procedures for patients allocated to FOBT, OC, or VC in order to inform the planning of a larger evaluative clinical trial.

\section{Methods}

\section{Participants and setting}

Patients age 50 to 70 years, identified from rosters of 5 participating family practices in the greater Hamilton region of Ontario, Canada, were eligible to participate in this study. Patients were excluded if they: were unable to give informed consent; had a history of colorectal cancer, adenomatous polyp, or inflammatory bowel disease; had undergone sigmoidoscopy, $\mathrm{OC}$, or $\mathrm{VC}$ within the previous 3 years; had undergone FOBT screening in the previous year; or, if they had severe or terminal illness that would preclude any benefit from colorectal cancer screening. The McMaster University Faculty of Health Sciences/ Hamilton Health Sciences Research Ethics Board approved this study (file number 09-147) and all participants gave written informed consent.

\section{Study procedures}

Patients were first mailed a one page invitation letter on their family physician's letterhead to participate in a study of colorectal cancer screening. The letter stated that colon cancer can be cured if detected early through screening and that patients who choose to participate in the study would be assigned at random to receive either FOBT, OC, or VC. Interested patients were invited to call a central booking office to schedule an appointment with the study nurse in order to learn more about the study. During the appointment, the study nurse enrolled eligible patients into the study. Patients who did not respond to the initial mailing were contacted once more either with a mailed reminder or a telephone follow-up call [5].

At the time of enrolment, we randomized patients in a 1:1:1 ratio using a parallel group design to colorectal screening with FOBT, OC, or VC. We used random variable block sizes and stratified by family physician practice and family history of colorectal cancer. Family history was obtained from an interview with the patient at the time of enrolment by the study nurse and was defined as a history of colorectal cancer or adenomatous polyps in a first-degree relative (parent, sibling, or child) at any age. We used an automated, centralized, telephonebased randomization service to maintain concealment of the computer generated allocation sequence. Patients allocated to FOBT were given the test kits along with educational materials and instructions for completion at the time of enrolment. Patients allocated to VC or OC were booked for these procedures using the conventional booking procedures for these investigations at our institution.

FOBT was performed by the patient at home using the guaiac-based FOBT kit recommended by the provincial Ontario Colon Cancer Check screening program (hema-screen, Immunostics Inc., Ocean, NJ, USA). Patients submitted the completed FOBT kit to a community laboratory and test results were sent to the patient's family physician and the study methods center.

Patients randomized to $\mathrm{OC}$ took a bowel preparation regimen the day before the procedure, consisting of: four $5 \mathrm{mg}$ tablets of bisacodyl, one sachet of sodium picosulfate $10 \mathrm{mg}$, magnesium oxide $3.5 \mathrm{~g}$, and citric acid $12 \mathrm{~g}$ (Picosalax, Ferring Inc., Toronto, Ontario, Canada) dissolved in $150 \mathrm{~mL}$ of water, and drinking at least $1 \mathrm{~L}$ of clear fluid. Patients took a second sodium picosulfate sachet dissolved in $150 \mathrm{~mL}$ of water and drank at least $1 \mathrm{~L}$ of clear fluid at bedtime. During the procedure, patients received intravenous sedation with midazolam and fentanyl as needed, with typical dosages of $3 \mathrm{mg}$ and 100 micrograms respectively, and received continuous monitoring of heart rate and oxygen saturation with a pulse oximeter. Decisions to biopsy suspicious lesions or perform polypectomy were left to the discretion of the endoscopist. OC examinations were performed by staff gastroenterologists at our academic health sciences centre who were fully licensed for independent practice by the regulatory body in our province.

Patients randomized to $\mathrm{VC}$ took a bowel preparation regimen (same regimen as the $\mathrm{OC}$ arm). Two hours before VC, patients received a small volume of oral contrast, which consisted of $20 \mathrm{~mL}$ of diatrizoate meglumine/diatrizoate sodium (Gastrografin, E-Z-EM Canada, Anjou, Quebec, Canada) diluted in $200 \mathrm{~mL}$ of water to tag any residual feces in the bowel $[6,7]$. Once positioned in the computed tomography scanner, patients were given a bowel antispasmodic, $20 \mathrm{mg}$ of hyoscine butylbromide (or, if contraindicated, $0.1 \mathrm{mg}$ of glucagon) intravenously. A mechanical insufflator was used to insufflate carbon dioxide through a rectal tube to maintain a steady pressure of 20 to $23 \mathrm{mmHg}$. Single breath-hold computed tomographs were 
acquired without intravenous contrast with the patient in the supine and prone positions (total radiation dose 200 milliamperes-seconds). Images were reviewed by radiologist promptly to determine whether follow-up OC for polypectomy or biopsy was needed. According to accepted practice at the time of the study, polyps were only reported if greater than $6 \mathrm{~mm}$ in size.

Abnormalities found on any of the screening tests were communicated to the participant's family physician, and decisions about the follow-up of abnormal test results were left to the discretion of the family physician. Research staff contacted patients by telephone once at 6 months after the study intervention to ascertain whether the patient had undergone the study intervention and to document whether crossover to another study arm had occurred. At 6 months follow-up, research staff also reviewed patients' medical records (at Hamilton Health Sciences, where the screening OC and VC procedures were performed, and at the participating family physician offices) to confirm the results of the index screening test and to collect data about further testing that was done to follow-up on findings from the index screening test. Patients, care providers, and outcome assessors were not blinded to the study intervention.

\section{Outcomes}

The primary outcome was completion of the initial colorectal screening intervention to which the patient was allocated. In other words, FOBT was considered complete if the patient returned the FOBT kit to the lab, regardless of whether they underwent follow-up OC if the FOBT test was positive. Similarly, VC was considered complete if the patient underwent the VC examination, regardless of whether they underwent follow-up OC based on VC findings. Secondary outcomes were: the proportion of patients who crossed over to another arm of the study; results of the index screening test; for patients allocated to $\mathrm{VC}$, the proportion of patients who received same or next day OC to follow-up results found on the index VC test; and the proportion of patients in whom colorectal adenoma or invasive carcinoma was found at 6 months follow-up.

\section{Sample size calculation}

Using a conservative assumption that $50 \%$ of patients in a given arm would complete their allocated screening test (a proportion of $50 \%$ would yield the widest $95 \%$ confidence interval) we required 196 patients to be enrolled in each arm to estimate the proportion of patients who complete screening with a $95 \%$ confidence interval of plus or minus $7 \%$. Therefore, we sought to enroll 200 patients in each arm. This sample size would also have permitted detection of an absolute difference of $13 \%$ or more in the primary outcome between any 2 arms with a significance level of 0.05 and $80 \%$ power. However, the trial was stopped early due to lack of ongoing funding.

\section{Statistical analysis}

Baseline clinical characteristics, primary and secondary outcomes are presented using descriptive statistics (i.e., mean and standard deviation for continuous variables and proportions for categorical variables). We compared the proportion of patients who completed their allocated screening test (primary outcome) across the 3 arms using a chisquared test. Statistical tests were 2-tailed using a significance level of 0.05 . Analyses of the primary outcome were conducted according to the intention-to-treat principle.

\section{Results}

\section{Study cohort}

Participants were enrolled between March 23, 2010 and August 11, 2010 after which the trial was stopped early due to lack of funding. A total of 198 participants were enrolled, of whom 67 were allocated to FOBT, 66 to OC, and 65 to VC (Fig. 1). Baseline characteristics of participating patients were similar across the three groups (Table 1).

\section{Completion of allocated colorectal cancer screening procedures}

The allocated screening procedure was completed by 43 (64 \%) subjects allocated to FOBT (95\% confidence interval [CI], $52 \%$ to $75 \%), 53$ (80\%) subjects allocated to OC (95\% CI, $69 \%$ to $88 \%)$, and 50 (77\%) subjects allocated to VC $(95 \% \mathrm{CI}, 65 \%$ to $85 \%)(P=0.08)$ (Fig. 1). Non-adherence to the allocated screening procedure was due to non-completion of the test, with the exception of 2 subjects who crossed over to another arm (1 crossover from FOBT to OC arm; 1 crossover from VC to OC arm). No subjects were lost to follow-up for the primary outcome. No subjects experienced complications of virtual or optical colonoscopy (e.g., bowel perforation).

\section{Outcomes after colorectal cancer screening}

Of the 43 subjects who underwent FOBT, 37 (86 \%) tested negative, 4 (9\%) had indeterminate results, and 2 (5\%) tested positive; 1 of the latter 2 subjects went on to have a follow-up OC which did not reveal any polyps or mass lesions.

Of the 55 subjects who received $\mathrm{OC}$ as their index screening test (including 2 crossovers from other arms), $22(40 \%)$ subjects had at least one polyp, most of which were $5 \mathrm{~mm}$ or less in size, and 12 subjects had at least one colorectal adenoma, one of which had evidence of high grade dysplasia (Table 2).

Of the 50 subjects who received VC, 8 (16\%) had at least one polyp greater than $5 \mathrm{~mm}$ in size (since smaller polyps were not reported) and $5(10 \%)$ underwent follow-up $\mathrm{OC}$ to further investigate findings seen on $\mathrm{VC}$ 


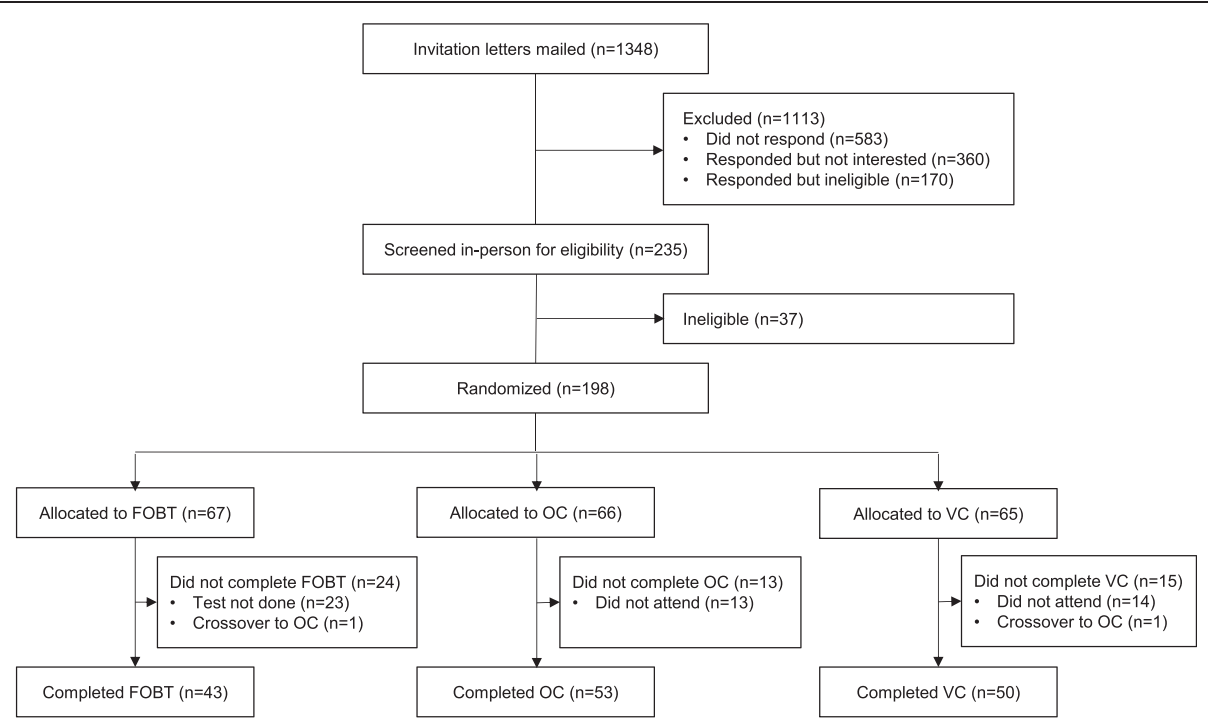

Fig. 1 CONSORT study flow diagram

(Table 3). Of the 5 subjects who underwent follow-up $\mathrm{OC}$, colorectal adenomas were found in 2 subjects, none of which had evidence of high-grade dysplasia. After VC, follow-up OC was able be performed on the same or next day in 3 of the 5 subjects who were referred for follow-up OC.

In summary, at 6 months follow-up, colorectal adenoma(s) were detected in $0(0 \%)$ subjects allocated to FOBT, 12 (18 \%) subjects allocated to OC, and 2 (3\%) subjects allocated to VC; one subject in the OC arm had histological evidence of high-grade dysplasia. None were diagnosed with colorectal cancer.

\section{Discussion}

In this pilot, randomized controlled trial of colorectal cancer screening, adherence to assigned colorectal cancer screening was $64 \%, 77 \%$, and $80 \%$ for patients allocated to FOBT, VC, and OC, respectively. Although the trial was stopped early, the findings may be useful to investigators planning randomized controlled trials of colorectal cancer screening in a primary care setting.

Our findings that $20 \%$ to $36 \%$ of patients did not complete their assigned screening test has several implications for a future evaluative trial. If the goal of a future trial were to assess the effect of these colorectal cancer

Table 1 Baseline characteristics of study participants

\begin{tabular}{|c|c|c|c|c|}
\hline Characteristics & $\begin{array}{l}\text { FOBT } \\
(N=67)\end{array}$ & $\begin{array}{l}\text { Optical Colonoscopy } \\
(N=66)\end{array}$ & $\begin{array}{l}\text { Virtual Colonoscopy } \\
(N=65)\end{array}$ & $P^{*}$ \\
\hline Age, years, mean (SD) & $58.7(5.4)$ & $58.4(5.6)$ & $59(5.6)$ & 0.77 \\
\hline Female & $31(46)$ & $25(38)$ & $38(58)$ & 0.06 \\
\hline Colorectal cancer in first degree relative(s) & $9(13)$ & $9(14)$ & $8(12)$ & 0.97 \\
\hline \multicolumn{5}{|l|}{ Signs or symptoms } \\
\hline Change in bowel habit & $4(6)$ & $1(2)$ & $1(2)$ & 0.23 \\
\hline Blood in stool & $3(4)$ & $0(0)$ & $1(2)$ & 0.18 \\
\hline Abdominal pain & $0(0)$ & $0(0)$ & $0(0)$ & 1.00 \\
\hline$>10 \%$ weight loss in past 6 months & $0(0)$ & $0(0)$ & $0(0)$ & 1.00 \\
\hline Unexplained anemia & $0(0)$ & $0(0)$ & $0(0)$ & 1.00 \\
\hline \multicolumn{5}{|l|}{ Smoking history } \\
\hline Never & $36(54)$ & $34(51)$ & $29(45)$ & 0.63 \\
\hline Former & $27(40)$ & $25(38)$ & $30(46)$ & \\
\hline Current & $4(6)$ & $7(11)$ & $6(9)$ & \\
\hline
\end{tabular}

Data are reported as number (percent) unless otherwise indicated. FOBT, fecal occult blood testing

${ }^{*} P$ value is for comparison of means or proportions across the 3 groups using one-way analysis of variance or chi-squared tests, respectively 
Table 2 Findings on optical colonoscopy

\begin{tabular}{ll}
\hline & $\begin{array}{l}\text { Patients with optical } \\
\text { colonoscopy as index } \\
\text { screening test }\end{array}$ \\
$(\mathrm{N}=55)$
\end{tabular}

Data are reported as number (percent) unless otherwise indicated

${ }^{a}$ includes 53 subjects originally allocated to $\mathrm{OC}$ and 2 subjects who crossed

over to OC from other arms

bas visualized on OC; polyp size data missing for 2 subjects

${ }^{c} 1$ of these subjects had an adenoma with high grade dysplasia

screening interventions under ideal conditions (i.e., efficacy), then our findings suggest that modification of our study procedures and a greater commitment of resources would be needed to increase adherence rates in a future clinical trial. In contrast, if the aim of a future trial were to evaluate the effectiveness (not efficacy) of colorectal cancer screening under conditions more reflective of usual practice, as was our intent, our findings provide useful estimates of anticipated adherence to FOBT, VC, and VC under typical conditions, since we did not provide any additional reminders for participants to complete their assigned screening procedures beyond usual booking or testing procedures. In particular, these estimates of test completion would be necessary to inform sample size calculations for a larger evaluative trial since non-completion of screening procedures would dilute any effect of the screening interventions on clinical outcomes such as colorectal cancer mortality.

Our observed adherence to FOBT of $64 \%$ is similar to that observed in the original trials of FOBT screening from the 1980s which documented adherence of $60 \%$ and $67 \%[8,9]$. In our trial, adherence was lower in the FOBT arm compared to the $\mathrm{OC}$ and VC arms, but the difference was not statistically significant. Other recent randomized controlled trials have reported data about screening completion rates. In one trial, participation rates were similar regardless of screening modality
Table 3 Findings on virtual colonoscopy

\begin{tabular}{ll}
\hline & $\begin{array}{l}\text { Patients with virtual } \\
\text { colonoscopy as index } \\
\text { screening test } \\
(N=50)\end{array}$ \\
\hline Subjects with polyp(s) ${ }^{a}$ & $8(16)$ \\
Largest polyp $6 \mathrm{~mm}$ to $9 \mathrm{~mm}$ & 7 \\
Largest polyp $10 \mathrm{~mm}$ or greater & 1 \\
Distribution of polyps by size, $\mathrm{n}^{\mathrm{a}}$ & \\
6 mm to 9 mm & 8 \\
10 mm or greater & 1 \\
Patients who underwent follow-up optical & 5 (10) \\
colonoscopy & \\
Distribution of polyps by histology, $\mathrm{n}$ & 3 \\
Sessile serrated adenoma & 2 \\
Patients with colorectal adenoma(s) & 0 \\
Patients with invasive carcinoma & \\
\hline Data are reported as number (percent) unless otherwise indicated \\
anly polyps greater than 5 mm in size were reported (see Methods)
\end{tabular}

(30.1\% for FOBT delivered by mail, $28.1 \%$ for FOBT delivered by a general practitioner or screening facility, and $28.1 \%$ for once-only sigmoidoscopy) [10]. In another clinical trial, a statistically significant difference in participation rates of $34.2 \%$ for fecal immunochemical testing versus $24.6 \%$ for optical colonoscopy was observed [11]. However, participation rates in these two trials are difficult to compare directly to ours since they randomized patients identified from population registries before obtaining informed consent for participation in the study, whereas we did not randomize patients to a screening intervention until after they gave consent to participate in the study. Our estimates of adherence are most applicable to patients who respond to invitations from their primary care physician to participate in colorectal cancer screening, agree to participate in a clinical trial, and then undergo allocated screening under usual conditions.

Our study has some limitations. First, we did not collect data from patients about the reasons why they did not complete their allocated screening test (e.g., lack of interest, failure of appointment booking procedures), which could have provided additional information to improve completion rates in a future trial. Second, our study was stopped early due to lack of funding. As a result, the estimates of adherence rates are more imprecise than we originally sought and, because of the reduced statistical power, it is not clear whether the lower adherence rate we observed for patients allocated to FOBT compared to the $\mathrm{VC}$ and $\mathrm{OC}$ arms is a true difference. Nonetheless, even though our estimates of adherence rates were not as precise as we had wanted (our original sample size would have provided $95 \%$ confidence 
intervals of, at most, plus or minus $7 \%$ ), we were still able to achieve a precision of approximately plus or minus $10 \%$ with our reduced sample size. Moreover, we believe that it is important to fully report data from clinical trials that are stopped early for insufficient recruitment (due to lack of funding or other reasons), since the failure to do so threatens the integrity of clinical research, and since investigators have an ethical responsibility to trial participants, to funders, and to the scientific community to disseminate the findings of their research [12]. Finally, the overall participation rate (i.e., number of patients enrolled out of the total number invited) in colorectal cancer screening in our study was low $(198 / 1,348=15 \%)$. This may have been a constraint of our randomized design in which participating patients could not choose which screening procedure they would get. It is possible that patients willing to be enrolled in a clinical trial are different from those who agree to undergo colorectal cancer screening after it is offered by their usual care provider in routine clinical practice.

\section{Conclusions}

In this pilot randomized controlled trial of colorectal cancer screening reflective of usual testing conditions in a primary care setting, we found that $64 \%$ to $80 \%$ of subjects completed their allocated screening test. These findings may be of value to investigators planning clinical trials to evaluate the effectiveness of colorectal cancer screening in primary care settings.

\section{Abbreviations}

Cl: confidence interval; FOBT: fecal occult blood testing; OC: optical colonoscopy; VC: virtual colonoscopy.

\section{Competing interests}

The authors declare that they have no competing interests.

\section{Authors' contributions}

JY conceived and designed the study, analyzed and interpreted the data, and drafted the manuscript. JK helped design the study and critically revised the manuscript. PV helped design the study and critically revised the manuscript. PM helped design the study and critically revised the manuscript. $C L$ analyzed and interpreted the data, and drafted the manuscript. All authors read and approved the final manuscript.

\footnotetext{
Acknowledgements

This study was funded by the Division of General Internal Medicine, McMaster University, Hamilton, Canada; the Department of Medicine, McMaster University, Hamilton, Canada; the Department of Medicine, Hamilton Health Sciences, Hamilton, Canada; a research grant from the Hamilton Health Sciences New Investigator Fund; and a research grant from the Juravinski Cancer Centre Foundation. Picosalax bowel preparation was provided by Ferring, Inc., Toronto, Ontario, Canada. The funders had no role in the design of the study, the collection, analysis, and interpretation of data, the writing of the manuscript, or the decision to submit the manuscript for publication. The authors thank Gordon Guyatt for sharing his expertise during the development of the study protocol, Imelda Esporlas-Jewer for her assistance with study coordination and patient recruitment, and Arthur Wong and Sohail Mulla for their assistance with data collection.
}

\section{Author details}

'Department of Medicine, McMaster University, 1280 Main Street West, Room HSC-2C8, Hamilton, Ontario L8S 4K1, Canada. ${ }^{2}$ Department of Clinical Epidemiology \& Biostatistics, McMaster University, 1280 Main Street West, Room HSC-2C8, Hamilton, Ontario L8S 4K1, Canada. ${ }^{3}$ Faculty of Dentistry, Western University, Dental Sciences Building, Room 1003, London, Ontario N6A 5C1, Canada. ${ }^{4}$ Department of Radiology, Connolly Hospital Blanchardstown, Dublin, Ireland. ${ }^{5}$ Department of Radiology, McMaster University, 1280 Main Street West, Room 2S284, Hamilton, Ontario L8S 4K1, Canada.

Received: 27 February 2015 Accepted: 29 June 2015

Published online: 09 July 2015

\section{References}

1. International Agency for Research on Cancer. GLOBOCAN 2012: Estimated Cancer Incidence, Mortality and Prevlance Worldwide in 2012. http:// globocan.iarc.fr/Pages/fact_sheets_cancer.aspx.

2. Yang DX, Gross CP, Soulos PR, Yu JB. Estimating the magnitude of colorectal cancers prevented during the era of screening: 1976 to 2009. Cancer. 2014;120:2893-901.

3. Levin B, Lieberman DA, McFarland B, Andrews KS, Brooks D, Bond J, et al. Screening and surveillance for the early detection of colorectal cancer and adenomatous polyps, 2008: a joint guideline from the American Cancer Society, the US Multi-Society Task Force on Colorectal Cancer, and the American College of Radiology. Gastroenterology. 2008;134:1570-95.

4. Screening for colorectal cancer. U.S. Preventive Services Task Force recommendation statement. Ann Intern Med. 2008;149:627-37.

5. Wong AD, Kirby J, Guyatt GH, Moayyedi P, Vora P, You JJ. Randomized controlled trial comparing telephone and mail follow-up for recruitment of participants into a clinical trial of colorectal cancer screening. Trials. 2013;14:40.

6. Johnson $\mathrm{CD}$, Chen MH, Toledano AY, et al. Accuracy of $C T$ colonography for detection of large adenomas and cancers. N Engl J Med. 2008;359:1207-17.

7. Kim DH, Pickhardt PJ, Taylor AJ, Heiken JP, Dachman A, Kuo MD, et al. CT colonography versus colonoscopy for the detection of advanced neoplasia. N Engl J Med. 2007;357:1403-12.

8. Hardcastle JD, Chamberlain JO, Robinson MH, Moss SM, Amar SS, Balfour TW, et al. Randomised controlled trial of faecal-occult-blood screening for colorectal cancer. Lancet. 1996;348:1472-7.

9. Kronborg O, Fenger C, Olsen J, Jorgensen OD, Sondergaard O. Randomised study of screening for colorectal cancer with faecal-occult-blood test. Lancet. 1996;348:1467-71.

10. Segnan N, Senore C, Andreoni B, Arrigoni A, Bisanti L, Cardelli A, et al. Randomized trial of different screening strategies for colorectal cancer: patient response and detection rates. J Natl Cancer Inst. 2005;97:347-57.

11. Quintero E, Castells A, Bujanda L, Cubiella J, Salas D, Lanas Á, et al. Colonoscopy versus fecal immunochemical testing in colorectal-cancer screening. N Engl J Med. 2012;366:697-706.

12. Kasenda B, Von EE, You J, Blümle A, Tomonaga Y, Saccilotto R, et al. Prevalence, characteristics, and publication of discontinued randomized trials. JAMA. 2014;311:1045-51.

\section{Submit your next manuscript to BioMed Central and take full advantage of:}

- Convenient online submission

- Thorough peer review

- No space constraints or color figure charges

- Immediate publication on acceptance

- Inclusion in PubMed, CAS, Scopus and Google Scholar

- Research which is freely available for redistribution 